\title{
Hypertensive crisis of a patient with undiagnosed paraganglioma
}

\author{
Kyu Bum Cho, Jeong Seok Lee, Jung Ha Lee, Dae Hun Yun, Cheon Hee Park \\ Department of Anesthesiology and Pain Medicine, Kwangju Christian Hospital, Gwangju, Korea
}

Received May 30, 2019

Revised June 3, 2019

Accepted June 5, 2019

Corresponding author

Cheon Hee Park

Department of Anesthesiology and

Pain Medicine, Kwangju Christian

Hospital, 37, Yangnim-ro, Nam-gu,

Gwangju 61661, Korea

Tel: +82-62-650-5150

Fax: + 82-62-650-5381

E-mail: jkb6257@naver.com

ORCID:

https://orcid.org/0000-0001-7986-8416

\begin{abstract}
Paragangilomas are uncommon catecholamine-secreting neuroendocrine tumors that derived from the autonomic nervous system. Patients with paraganglioma may develop cardiovascular complication such as severe hypertensive crisis during anesthesia or surgery. It can lead to a life threatening condition if undiagnosed preoperatively or not adequately prepared. Paragangiloma and gastrointestinal stromal tumors (GISTs) may have a similar radiographic appearance. If there are no histologic confirmation and clinical manifestation of catecholamine excess, paragangliomas may be mistaken of GISTs. We report the case of a 56-year-old woman who developed hypertensive crisis during anesthesia for GISTs excision.
\end{abstract}

Keywords: Paraganglioma; Gastrointestinal stromal tumor; Pheochromocytoma; Anesthesia

\section{INTRODUCTION}

Paraganglioma is a rare catecholamine-producing tumor derived from the chromaffin cell of sympathetic ganglion, also called extra-adrenal pheochromocytoma. It can be found anywhere along the sympathetic trunk from the skull base to the neck, pelvis, prostate, and bladder [1,2]. The clinical manifestations of paraganglioma may vary from asymptomatic to catecholamine-induced symptoms such as headache, palpitation and sweating. Hypersecretion of catecholamine can also lead to life-threatening conditions such as uncontrolled hypertension, arrhythmia, cardiomyopathy, myocardial infarction, and pulmonary edema [2]. Due to the its rarity and non-specific signs and symptoms, it is rarely diagnosed before surgery or is found incidentally during sur- gery. Unexpected excessive release of catecholamine during surgery can make anesthetic care difficult, requiring appropriate management.

Extraluminal perigastric mass is often seen as gastrointestinal stromal tumor (GIST) or soft tissue sarcomas without pathologic confirmation [3]. With absence of pathologic diagnosis and catecholamine-induced symptoms, paraganglioma can also be misdiagnosed as GIST due to its similarity in radiologic evaluation. In this study, a case of hypertensive crisis in paraganglioma patients who were misdiagnosed as GIST is reported.

\section{CASE REPORT}

A 56-year-old female patient with a height of $156 \mathrm{~cm}$ and 


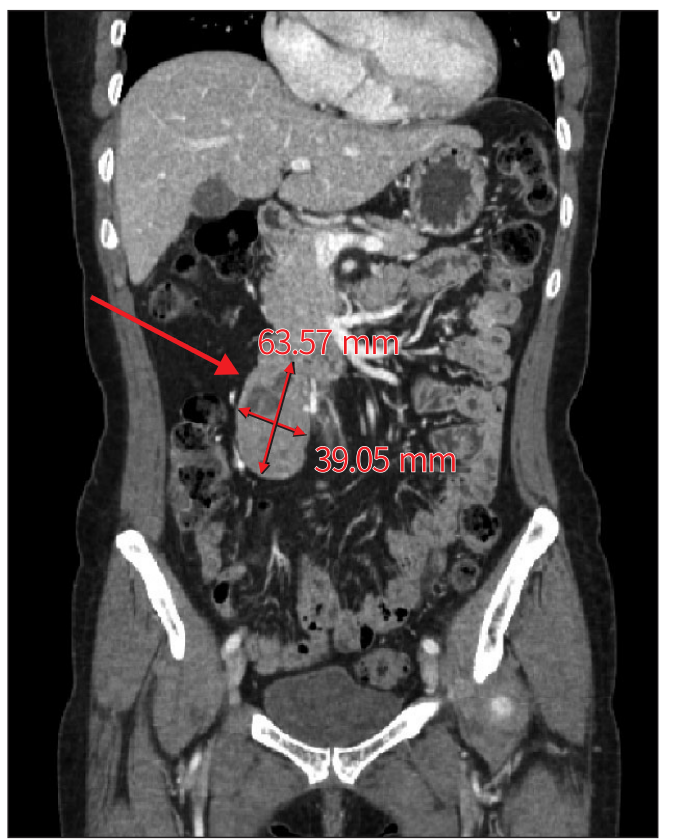

Fig. 1. Contrast-enhanced CT image shows $3.9 \times 6.3 \mathrm{~cm}$ well defined heterogeneous enhancing mass in duodenal $3^{\text {rd }}, 4^{\text {th }}$ portion (indicated by the arrow).

a body weight of $56 \mathrm{~kg}$ who had improvements after treatment influenza A in a private clinic, visited our clinic for precise evaluation of amylase elevation found at admission. Abdominal computed tomography image revealed a $39 \times 63$ mm GIST between the third and fourth portions of duodenum and no lesion was found in upper gastrointestinal endoscopy within the observable range. Laparoscopic wedge resection was planned for surgical removal of the tumor (Fig. 1).

There was no current medication. Preoperatively performed electrocardiogram showed normal sinus rhythm at 75 beats per minute and there was no abnormality on chest $\mathrm{X}$-ray. The blood test was normal except for elevation of amylase $913 \mathrm{U} / \mathrm{L}(7-60 \mathrm{U} / \mathrm{L})$. On the day of the operation, the blood pressure (BP) measured at the ward was 120/80 $\mathrm{mmHg}$, the heart rate (HR) 64 beats/min, the respiratory rate 18 breaths $/ \mathrm{min}$, and the temperature $36.5^{\circ} \mathrm{C}$. Famotidine $20 \mathrm{mg}$ and glycopyrorate $0.2 \mathrm{mg}$ were administered for preanesthetic medication. After the entrance of operation room, $\mathrm{BP}$ was measured to be $177 / 106 \mathrm{mmHg}$, HR to be 78 beats/min, oxygen saturation to be $99 \%$, and electrocardiogram showed normal sinus rhythm. For anesthetic induction, the patient was intubated after muscle relaxation with administration of profopol $120 \mathrm{mg}$, fentanyl $100 \mathrm{mcg}$, and rocuronium $40 \mathrm{mg}$. After intubation, $1 \mathrm{~L} / \mathrm{min}$ of oxygen and $2 \mathrm{~L} / \mathrm{min}$ of air were mixed to maintain an inhaled oxygen concentration of 50\%. For maintenance, desflurane 6.0 vol\% and remifentanil $0.02-0.06 \mathrm{mcg} / \mathrm{kg} / \mathrm{min}$ was administered. The end tidal partial pressure of carbon dioxide was 35-38 mmHg and the bispectral index (BIS) was between 41-50. The patient remained hemodynamically stable during anesthetic induction, endotracheal intubation, and skin incision.

At 25 minutes after starting the operation, as the operator initially operated the tumor, the BP increased to $266 / 153$ mmHg and the HR to $110-130$ beats/min with a bigeminy pattern. The surgery was immediately interrupted, and intravenous bolus injections of $1 \mathrm{mg}$ of nicardipine and $5 \mathrm{mg}$ of labetalol were given, followed by an increase in the depth of anesthesia with 9.0 vol\% of desflurane and a continuous infusion of remifentanil at a rate of $0.2 \mathrm{mg} / \mathrm{kg} / \mathrm{min}$. For accurate hemodynamic monitoring, a 20-gauge catheter was placed in the left radial artery and $5 \mathrm{mg}$ of labetalol was added for HR control. The BP remained at 212/124 mmHg, so a continuous intravenous infusion of nicardipine at a rate of $2-5 \mathrm{mcg} / \mathrm{kg} / \mathrm{min}$ was started. Then, the BP was decreased to $117 / 82 \mathrm{mmHg}$ and the operation was resumed with a systolic BP of 110-120 mmHg and a HR of 70-80 beats/min. When the tumor was completely removed, the BP gradually decreased and the continuous administration of nicardipine was stopped. With BP of 80 / $60 \mathrm{mmHg}$, and $4 \mathrm{mg}$ ephedrine was injected and the crystalloid was loaded.

The total anesthesia time including the operation time was 165 minutes. The estimated blood loss volume was $200 \mathrm{~mL}, 850 \mathrm{~mL}$ of crystalloid was administered, and 250 $\mathrm{mL}$ of urine output was recorded. When the patient's vital signs were stable, the train-of-four count was 3 , so $2 \mathrm{mg} /$ $\mathrm{kg}$ of sugammadex was administered intravenously. When the train-of-four ratio was 0.9 , the patient was fully awake and extubated. After confirming the hemodynamically stable condition, the patient was transferred to general ward from the recovery room. On the fifth postoperative day, the patient was discharged without complications. The microscopic findings of the resected mass revealed that the tumor cells with abundant cytoplasm formed a small group of cells surrounded by the interstitial tissue (Fig. 2). These cells were strongly positive for chromogranin in immunohistochemical staining, showing the chromaffin affinity and thereby, diagnosed for paraganglioma (Fig. 3). 


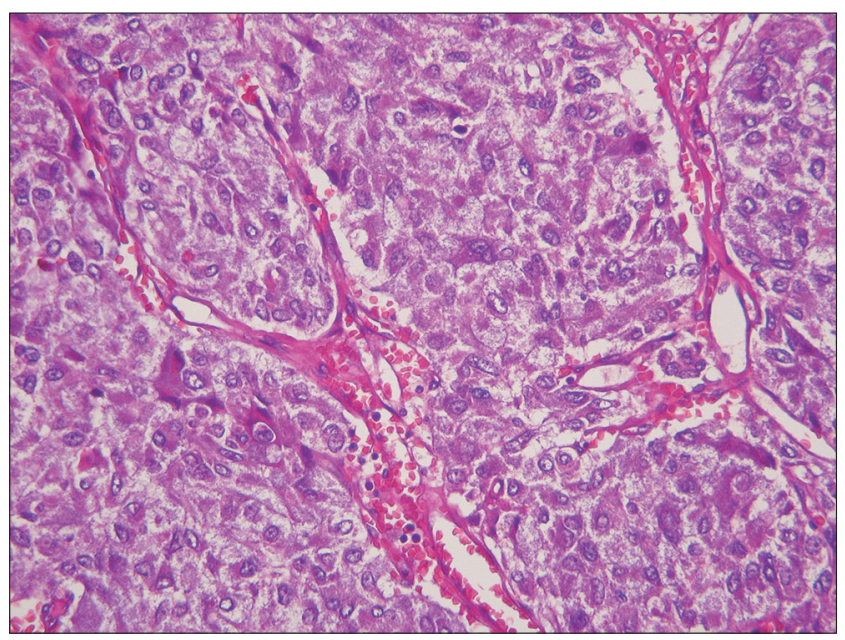

Fig. 2. Histologic examination shows well defined nests of tumor cells ("Zellaben") separated by fibrous septa. The individual cells have abundant granular cytoplasm and pleomorphic nuclei (×200, Hematoxylin \& Eosin).

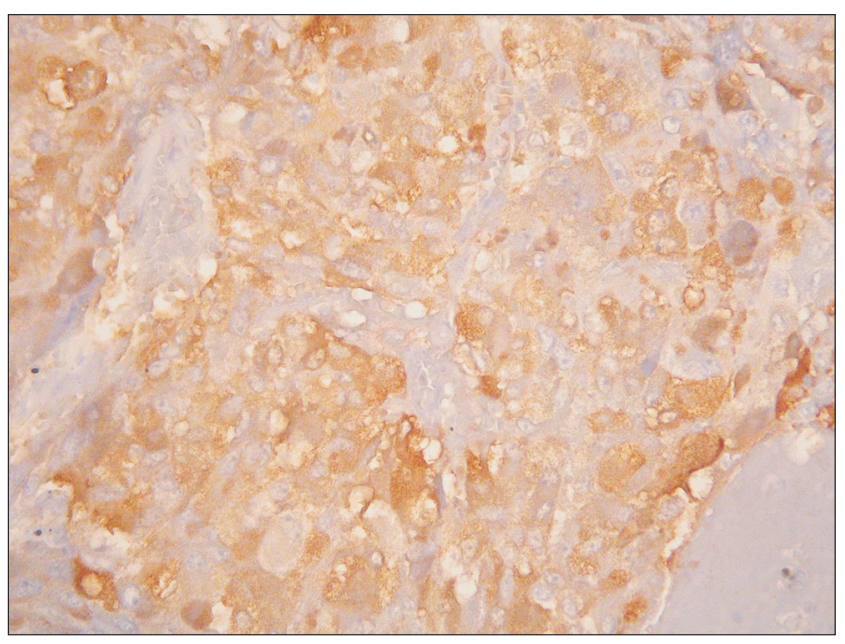

Fig. 3. The tumor cells reveal immunoreactivity for chromogranin $(\times 200$, Chromogranin).

\section{DISCUSSION}

Paraganglioma is a chromaffin cell tumor rarely originating from neural crest cells. When the tumor is derived from adrenal medulla, it is called pheochromocytomas, while it is derived from extra-adrenal site, it is called extra-adrenal pheochromocytomas [1]. Tumor can be formed anywhere from the cranial base to the epididymis and sympathetic paragangliomas are mostly functional, mainly found in the abdomen, while parasympathetic paragangliomas are mostly nonfunctional and mainly found in the head and neck [4]. Paraganglioma accounts for 10 to $18 \%$ of all chro- maffin-related tumors [1]. About 30\% of paragangliomas are hereditary. It is highly related to hereditary syndromes such as neurofibromatosis type 1, von Hippel-Lindau disease, Multiple endocrine neoplasia (MEN) type 1, 2, and succinate dehydrogenase mutation [2]. Therefore, genetic analysis is recommended in paraganglioma patients [3]. Unlike pheochromocytoma, which has a $10 \%$ probability of malignancy, paraganglioma is malignant in 15 to $35 \%$ [1,3]. The radiologic findings of large size, heterogeneity, uneven borderline, and distant metastasis may imply malignancy but, there is no clear diagnostic criteria [5].

Patients with paraganglioma manifest signs due to catecholamine excess. Headache, sweating, and palpitations are the typical triad symptoms, but patients also can be asymptomatic, or have various clinical manifestations such as tremor, pallor, anxiety, weight loss, hyperglycemia, stroke, cardiomyopathy $[1,4,6$. Diagnosis may be delayed or overlooked due to its rarity and non-specific clinical features. Mostly, patients are initially examined due to the symptoms associated with a mass effect, or tumors are found incidentally on a CT or MRI scan. Only 20\% of paragangliomas are found because of the hyper-function of the tumor itself [7].

Radiologic evaluation such as CT and MRI are useful for diagnosis. The sensitivity of CT for diagnosis of abdominal paraganglioma is about $90 \%$, and ${ }^{123}$ I-MIBG scintigraphy may also help diagnosis [4]. 24-hour urine catecholamine and metabolite measurements are useful for differential diagnosis of paraganglioma. Because the half-life of norepinephrine and epinephrine is very short, it is effective to measure the inactivated metabolites, normetanephrine and metanephrine. The sensitivity and specificity of the 24-hour urine metanephrine test are $89.9 \%$ and $99 \%$, respectively [1].

Serum amylase elevation has many causes besides pancreatitis and tumors. In acute abdomen and pancreatitis patients, incidental pheochromocytoma has been reported to be found, but finding the cause to an isolated elevated serum amylase in an asymptomatic patient is challenging. In this case, a one-year follow-up examination after tumor resection still showed an elevated serum amylase of $738 \mathrm{U} / \mathrm{L}$ (54-168 U/L), and further differential diagnosis seems necessary.

GIST is a benign gastric tumor that is characterized by relatively large size $(>5 \mathrm{~cm})$, heterogeneous enhancement (84\%) and central necrosis (37\%) [8]. A large sized paragan- 
glioma upper gastrointestinal tract is often misdiagnosed as a neurogenic or mesenchymal tumor, such as GIST or soft tissue sarcoma, because there are no specific signs due to catecholamine excess and resemblance in imaging studies [9].

In the present case, the tumor was located near the stomach in the imaging and seemed to be a typical GIST. In addition, there was no signs of catecholamine hypersecretion, being asymptomatic, and the patient had no family history. Therefore, it is thought that surgical resection can be done without any problems and unfortunately, any further diagnostic evaluation including preoperative biopsy was not performed. The blood pressure at the arrival at the operation room was found to be slightly high due to preoperative anxiety, but there was no problem found in the process of induction of anesthesia, intubation, and skin incision. However, at the moment of the surgeon operating the tumor, the patient had a hypertensive crisis and showed hypotension after tumor removal. It is thought to be due to the sudden release of excess catecholamine contained in paraganglioma, which is mistaken for GIST. Most report considerable hemodynamic stability during induction and maintenance of anesthesia using inhalation anesthesia and opioids, but experience a hypertensive crisis at the moment the mass is maneuvered [7,10]. In a case described in 2009, Lowenthal et al. [6] reported that the tumor located near the stomach was mistaken for GIST, but they experienced a hypertensive crisis as soon as the initial operational step for tumor removal was performed for resection. There are reports that paraganglioma was found not only in the duodenum but also in the liver, urethra, cerebellopontine angle, cauda equine, and larynx [11]. Therefore, the doctors and anesthesiologists should be aware of the possibility of paraganglioma even in unexpected sites during surgery.

Preoperative treatment of paraganglioma patients is important. Catecholamine excess can result in perioperative hypertension and arrhythmia, which may lead to serious hemodynamic changes and increase mortality. Obtaining adequate circulating plasma volume and a high salt diet can be helpful. In order to control the circulating catecholamine effect, $\alpha$ blockers (phenoxybenzamine, prazosin, doxazosin) should be used and beta blockers (atenolol, propranolol) should be added in case of arrhythmia caused by $\alpha$ blocker. These efforts can reduce the perioperative mortality from $40-60 \%$ to $0-6 \%$ [2,12-14]. Some drugs, such as dopamine-receptor antagonists, appear to consistently produce adverse reactions. The stimulation of dopamine type- 2 receptors on sympathetic nerve endings inhibits the release of catecholamines. Metoclopramide blocks this inhibition and may act as potent trigger of severe catecholamine release in the presence of a pheochromocytoma, which can result in acute cardiogenic shock, as it may have in this patient. Available literature recommends avoiding certain drugs in patients with a suspected pheochromocytoma, including opiates, blockers of catecholamine reuptake like tricyclic antidepressants and cocaine, sympathomimetic amines like tyramine, decongestants, amphetamines, corticosteroid hormones, and paralytics with vagolytic properties like pancuronium and atracurium [15]. All agents that cause histamine release should be avoided. The depolarizing agent, succinylcholine, has the potential for causing catecholamine surges from the muscle fasciculations that it produces which can mechanically compress the tumor, in addition to stimulation of the autonomic ganglia, both of which can be detrimental [16].

In patients with hypertensive crisis during the operation of paraganglioma, nitroprusside, nitroglycerin, phentolamine, $\beta$-blocker (esmolol), and calcium channel blocker (nicardipine) may be used [14]. In addition, the use of short-acting agents is preferable to long-acting agents because the blood pressure and heart rate can vary widely $[1,17]$. In this case, we could control the blood pressure by continuous administration of nicardipine in the hypertensive crisis and proceed with the operation by using beta blocker for the tachyarrhythmia. It has been reported that nicardipine is effective in the anesthesia care in patients with pheochromocytoma due to its potent vascular relaxation effect and catecholamine secretion inhibition effect [18]. The selection of inhalation anesthetic is still under discussion while some report that desflurane should be avoided due to its sympathetic stimulation effect and others assert that it is an option for use [16,19].

Hypotension may occur after tumor removal due to inadequate vascular volume, remnant effect of alpha blocker, bleeding. For correction of hypotension, proper fluid therapy should firstly be considered and vasopressors should be used if necessary [14]. In this case, hypotension was observed after tumor removal, and normal blood pressure 
could be maintained by loading of crystalloid fluid and intravenous injection of ephedrine.

In conclusion, paraganglioma is not easy to diagnose if a patient has no family history, is asymptomatic, or has ambiguous symptoms. Therefore, in the case of operation for incidentaloma in the chest and abdomen including GIST, paraganglioma should be suspected if the patient is suffering from hemodynamic instability such as uncontrolled hypertension or tachycardia, during the induction of anesthesia and surgical operation. Appropriate preoperative and intraoperative managements are essential for a better prognosis.

\section{ACKNOWLEDGMENTS}

I would like to express my gratitude to Jae-hoon Jeong, M.D. and Jae-hong Lee, M.D. for his contributions to the paper.

\section{CONFLICT OF INTEREST}

No potential conflict of interest relevant to this article was reported.

\section{REFERENCES}

1. Lee JA, Duh QY. Sporadic paraganglioma. World J Surg 2008;32:683-7.

2. Koumaras CH, Anagnostis P, Tzimou M, Giavanidis I, Gossios T, Antoniadis A, et al. Paraganglioma in a young patient with asymptomatic severe hypertension: a case report and review of the literature. Hippokratia 2010;14:300-2.

3. Somasundar P, Krouse R, Hostetter R, Vaughan R, Covey T. Paragangliomas-- a decade of clinical experience. J Surg Oncol 2000;74:286-90.

4. Renard J, Clerici T, Licker M, Triponez F. Pheochromocytoma and abdominal paraganglioma. J Visc Surg 2011;148:e40916.

5. Waguespack SG, Rich T, Grubbs E, Ying AK, Perrier ND, Ayala-Ramirez M, et al. A current review of the etiology, di- agnosis, and treatment of pediatric pheochromocytoma and paraganglioma. J Clin Endocrinol Metab 2010;95:2023-37.

6. Lowenthal MS, Sadow PM, Raut C, Metzler EC. Intraoperative diagnosis of a functional paraganglioma presenting as a gastrointestinal stromal cell tumor (GIST). J Clin Anesth 2009;21:57-60.

7. Han IS, Kim YS, Yoo JH, Lim SS, Kim TK. Anesthetic management of a patient with undiagnosed paraganglioma -a case report-. Korean J Anesthesiol 2013;65:574-7.

8. Sandrasegaran K, Rajesh A, Rushing DA, Rydberg J, Akisik FM, Henley JD. Gastrointestinal stromal tumors: CT and MRI findings. Eur Radiol 2005;15:1407-14.

9. Hayes WS, Davidson AJ, Grimley PM, Hartman DS. Extraadrenal retroperitoneal paraganglioma: clinical, pathologic, and CT findings. AJR Am J Roentgenol 1990;155:1247-50.

10. Shinn HK, Jung JK, Park JK, Kim JH, Jung IY, Lee HS. Hypertensive crisis during wide excision of gastrointestinal stromal cell tumor (GIST): Undiagnosed paraganglioma -A case report-. Korean J Anesthesiol 2012;62:289-92.

11. Toyota B, Barr HW, Ramsay D. Hemodynamic activity associated with a paraganglioma of the cauda equina. Case report. J Neurosurg 1993;79:451-5.

12. Reisch N, Peczkowska M, Januszewicz A, Neumann HP. Pheochromocytoma: presentation, diagnosis and treatment. J Hypertens 2006;24:2331-9.

13. Pacak K. Preoperative management of the pheochromocytoma patient. J Clin Endocrinol Metab 2007;92:4069-79.

14. Kinney MA, Narr BJ, Warner MA. Perioperative management of pheochromocytoma. J Cardiothorac Vasc Anesth 2002;16:359-69.

15. Sheinberg R, Gao WD, Wand G, Abraham S, Schulick R, Roy $\mathrm{R}$, et al. Case 1--2012. A perfect storm: fatality resulting from metoclopramide unmasking a pheochromocytoma and its management. J Cardiothorac Vasc Anesth 2012;26:161-5.

16. Ramakrishna H. Pheochromocytoma resection: current concepts in anesthetic management. J Anaesthesiol Clin Pharmacol 2015;31:317-23.

17. Plouin PF, Gimenez-Roqueplo AP. Pheochromocytomas and secreting paragangliomas. Orphanet J Rare Dis 2006;1:49.

18. Arai T, Hatano Y, Ishida H, Mori K. Use of nicardipine in the anesthetic management of pheochromocytoma. Anesth Analg 1986;65:706-8.

19. Domi R, Sula H. Pheochromocytoma, the challenge to anesthesiologists. J Endocrinol Metab 2011;1:97-100. 